\title{
Who care? virtual therapist to assist caregivers of stroke patients - a pilot study
}

\author{
Bryan Ping Ho Chung*, Cherry Chau Yee Yeung, Gloria Hiu Wing Lau, Michelle Yan Ki Tse, Tak \\ Man Lo, Titanic Fuk On Lau
}

Physiotherapy Department, Tai Po Hospital, Tai Po, New Territories, Hong Kong, China

Accepted on February 23, 2018

\section{Introduction}

Caregivers of stroke patients take over the roles of nurses, therapists and health workers once the patients were discharged home. Evidence show that caregiver training is an essential component of stroke rehabilitation to decrease hospital stay [1], enhance carer's quality of life [1], reduce burden of care, and reduce risk of injury to carers and patients [2-4]. Since traditional caregiver trainings are relied on direct therapist contact with caregivers within limited contact time, it is a challenge for therapists to ensure caregivers could remember the knowledge and skills. Recent evidence showed that educational video can improve performance accuracy [5] and knowledge of patients with stroke [6]. A pilot study of Virtual Therapist had been launched in inpatient stroke rehabilitation program of Tai Po Hospital by Physiotherapy Department. Virtual Therapist took advantages of recent advances in videosharing technologies via smart phone or tablets that allow caregivers of stroke patients to review the videos just like a virtual therapist is available for consultation anytime and anywhere.

\section{Objective}

Evaluate the feasibility and effectiveness of Virtual Therapist in enhancing knowledge, skills and perceived competence of caregivers of stroke patients as well as reducing the percentage of direct therapist contact time in caregiver training of stroke rehabilitation.

\section{Methodology}

Pre-discharge caregiver trainings for stroke patients were arranged once caregivers were available. The caregivers in control group received traditional caregiver training by therapists supplemented by educational booklets. The caregiver in Virtual Therapist group received Virtual Therapist which consisted of 13 videos (total 39 minutes) including knowledge of stroke, skill demonstrations of positioning, stretching, transfer and ambulation by physiotherapists. The videos were produced by a group of physiotherapists and digital video enthusiasts of Tai Po Hospital. Suitable videos were selected by physiotherapists according to patients' physical conditions. At the beginning of caregiver training sessions, supporting staff used tablets to present the videos to caregivers. When the caregivers finished watching the videos, physiotherapists contacted the caregivers and ensured their skills were up to standard. A brochure with QR codes of the videos was given to the caregivers so they could review the videos while practicing the skills in wards before patients were discharged or practicing the skills at home after patients were discharged.

Outcome measurements including knowledge score (0-10), skill performance score (0-5), perceived competence score (0-28) and percentage of direct therapist contact time in caregiver training (\%) were collected by therapists after each caregiver training session. The outcomes of control group and Virtual Therapist group were collected from Sep 2017 to Oct 2017 and Nov 2017 to Dec 2017 respectively.

\section{Results and Outcome}

A total of 50 caregivers were recruited. Control group had 26 caregivers of 26 stroke patients (18 male, 8 females, mean age 70.4). Virtual Therapist group had 24 caregivers of 24 stroke patients (17 male, 7 females, mean age 71.6). Compared the 2 groups, Virtual Therapist group had significant higher in knowledge score (7.1 vs. 3.6, $\mathrm{p}<0.001)$, skill performance score ( 4.8 vs. $4.0, p<0.005)$ and perceived competence score (22.1 vs. $20.3, \mathrm{p}<0.05)$ than control group. Percentage of direct therapist contact time in caregiver training in Virtual Therapist group had significant lower $(49 \%$ vs $99 \%, \mathrm{p}<0.001)$ than control group. Feedbacks from caregivers and therapists were positive. Over $95 \%$ of caregivers had smart phone and were able to use the brochure with QR codes to review the videos.

The results supported that Virtual Therapist through therapistdemonstrated video is feasible and could enhance knowledge, skills, and perceived competence of caregivers for stroke patients as well as reduce percentage of direct therapist contact time in caregiver training of stroke rehabilitation. The project had promising results and is now adapted to our daily routines.

\section{References}

1. Patel A, Knapp M, Evans A, et al. Training care givers of stroke patients: economic evaluation. BMJ 2004;328(7448): 1102.

2. McCullagh E, Brigstocke $\mathrm{G}$, Donaldson $\mathrm{N}$, et al. Determinants of caregiving burden and quality of life in caregivers of stroke patients. Stroke 2005;36(10):2181-6.

3. Pierce LL, Steiner V, Govoni A, et al. Two sides to the caregiving story. Top Stroke Rehabil 2007;14(2):13-20.

4. Hinojosa MS, Rittman M. Association between health education needs and stroke caregiver injury. J Aging Health 2009;21(7):1040-58.

5. Reo JA, Mercer VS. Effects of live, videotaped, or written instruction on learning an upper-extremity exercise program. Phys Ther 2004;84(7):622-33. 
Citation: Chung BPH, Yeung CCY, Lau GHW, et al. Who care? virtual therapist to assist caregivers of stroke patients - a pilot study J Phys Ther Sports Med 2018;2(1):15-16.

6. Denny MC, Vahidy F, Vu KY, et al. Video-based educational intervention associated with improved stroke literacy, self-efficacy, and patient satisfaction. PloS ONE 2017;12(3):e0171952.

\section{*Correspondence to:}

Bryan Ping Ho Chung

\section{Physiotherapy Department}

Tai Po Hospital, 11 Chuen On Road, Tai Po

New Territories, Hong Kong, China

E-mail: taipobryan@yahoo.com 